\title{
Importance des recombinaisons ARN dans l'évolution des lutéovirus
}

\author{
BA Chalhoub ${ }^{1 *}$, HD Lapierre ${ }^{2}$ \\ 1 INRA, génétique et amélioration des plantes; \\ 2 INRA, pathologie végétale, route de Saint-Cyr, F78026 Versailles cedex, France
}

(Reçu le 20 mai 1995 ; accepté le 4 juillet 1995)

\begin{abstract}
Résumé - Les lutéovirus ont été reconnus comme un groupe de phytovirus par l'ITCV (International Committee on Taxonomy of Viruses) en 1975. Ils ont en commun un certain nombre de propriétés comme la structure isométrique de leurs particules, leur restriction au tissu phloémique de la plante hôte et leur transmission obligatoire par puceron. Ils ont été initialement divisés en 2 sous-groupes, sur la base de leurs relations sérologiques et leurs effets cytopathologiques. Les séquences complètes ou partielles du génome de plusieurs lutéovirus ont été déterminées. En fonction de la région du génome considérée, des relations phylogénétiques différentes sont observées entre les lutéovirus et d'autres phytovirus. Ces observations suggèrent un rôle important des recombinaisons ARN dans l'évolution des lutéovirus. Des modèles d'évolution des lutéovirus par recombinaison ARN ainsi que des propositions sur la taxonomie de cet ensemble de virus sont discutés.
\end{abstract}

lutéovirus / recombinaison ARN / évolution / taxonomie

Summary - Importance of RNA recombinations in the evolution of luteoviruses. Luteoviruses were recognized as a plant virus group by the International Committee on Taxonomy of Viruses (ITCV) in 1975. They have a number of biological properties in common such as their isometric particles, their restriction to phloem tissue and their dependence on aphid vectors for transmission. They were initially separated into 2 distinct subgroups on the basis of their serological relatedness and cytopathological effects. During the last few years, complete genome sequences and organisations have been determined for many luteoviruses and, depending on the region of the genome, various relationships are observed between luteoviruses and other plant viruses. More sequence homologies are observed between luteoviruses, for the 3' half of their genome coding for the structural proteins (capsid, readthrough) and the putative movement protein (figs 1 and 2a). Luteoviruses differ mainly in the evolutionary origin of their RNA-dependent RNA polymerases (RdRp) encoded by the $5^{\prime}$ half of the genome (figs 1 and $2 b$ ). Thus, the RdRp of the subgroup 1 luteoviruses (PAV and MAV serotypes of the barley yellow dwarf virus (BYDV) and the soybean dwarf virus) show many similarities with those of the dianthoviruses, carmoviruses and tombusviruses (fig $2 b$ ). In contrast, subgroup 2 luteoviruses (potato leafroll virus, beet western yellow virus (BWYV), cucurbit aphid borne virus and the BYDV-RPV), have a sobemo-like polymerase (fig $2 \mathrm{~b}$ ). This dichotomy in sequence homology between the $5^{\prime}$ and the $3^{\prime}$ halves of their genome suggests an important role of RNA recombination in the evolution of luteoviruses. Mixed infections occur between luteoviruses and other plant viruses and are favourable situations for the generation of new virus entities. The newly generated viruses may have genomes composed of 2 taxonomically different RNA molecules, such as the ST9 strain of the BWYV or the pea enation mosaic enamovirus. They may also have genomes generated by RNA recombination. Subgroup 1 luteoviruses may have been generated by a RNA recombination event between a subgroup 2 luteovirus and a dianthovirus (fig 1). Additional RNA recombination events that occurred during luteovirus evolution could be deduced from sequence comparison of strains of the same luteovirus or different luteoviruses.

luteovirus / RNA recombination / evolution / taxonomy / aphid borne

\footnotetext{
* Correspondance et tirés à part
} 


\section{INTRODUCTION}

Les recombinaisons ARN constituent un des mécanismes clés impliqués dans la variabilité génétique et l'évolution des virus à ARN (Lai, 1992 ; Simon et Bujarski, 1994). Bien que ces phénomènes aient été expérimentalement démontrés pour de rares virus animaux (Lai, 1992), des phytovirus (Simon et Bujarski, 1994) et bactériophages (Palasingam et al, 1992), les analyses phylogénétiques et les comparaisons des séquences montrent que les recombinaisons ARN jouent probablement un rôle important dans leur évolution.

La comparaison des séquences génomiques entre plusieurs lutéovirus et d'autres phytovirus montre des phylógénies différentes en fonction de la région du génome considérée.

Dans cette synthèse, ón prendra pour support les comparaisons phylogénétiques pour souligner l'importance des recombinaisons ARN dans l'évolution des lutéovirus et discuter les conséquences que celles-ci pourraient avoir dans leur taxonomie.

\section{GÉNÉRALITÉS}

Les propriétés biologiques, écologiques, épidémiologiques, et sérologiques des lutéovirus ont été décrites dans de nombreux travaux (Rochow et Duffus, 1981 ; Johnstone et al, 1984 ; Casper, 1988 ; Waterhouse et al, 1988). Des revues récentes portant sur l'organisation des génomes des lutéovirus, les fonctions et les stratégies d'expression des différents gènes ou cadres ouverts de lecture (ORF ; d'open reading frame) ont été réalisées (Martin et al, 1990 ; Miller et al, 1994). Un bref rappel sur ces principales propriétés est décrit dans ces généralités.

\section{Nomenclature}

Les lutéovirus ont été reconnus comme un groupe des phytovirus par l'ITCV (International Committee on Taxonomy of Viruses) en 1975 (Shephered et al, 1976). Le nom de ce groupe est dérivé du mot latin luteus (jaune), en raison des symptômes de jaunissement déterminés par certains de ses membres dans leurs plantes hôtes.

\section{Structure des particules}

Les particules purifiées des lutéovirus ont une structure isométrique de $24-28 \mathrm{~nm}$ de diamètre qui renferment une molécule d'ARN d'environ $2 \times$ $10^{6} \mathrm{Da}$ (Casper, 1988).

\section{Biologie}

Les lutéovirus sont inféodés aux plantes herbacées. Ils sont confinés au tissu phloémique (tubes criblés, cellules compagnes, parenchyme phloémique) de la plante hôte (Gill et Chong, 1975). Leur transmission s'effectue obligatoirement par l'intermédiaire de pucerons selon le mode circulant (Casper, 1988 ; Waterhouse et al, 1988).

\section{Sérologie}

La plupart des lutéovirus sont reliés sérologiquement et ont été divisés à l'aide d'anticorps polyclonaux en 2 principaux sous-groupes ( $\mathrm{sg} 1$ et sg2) (Waterhouse et al, 1988).

\section{Organisation du génome}

Le génome des lutéovirus est composé d'une molécule ARN simple brin de polarité positive et d'environ 5600 à 5700 nucléotides. Cet ARN n'a pas une queue polyadénosine [poly (A)] ou des structures particulières à son extrémité 3 .

Selon l'organisation de leurs génomes et les homologies des séquences, les lutéovirus séquencés peuvent être divisés en 2 principaux sous-groupes (fig 1). Le sous-groupe 1 représenté par le sérotype PAV du virus de la jaunisse nanisante de l'orge (BYDV-PAV) (Miller et al, 1988), comprend aussi le BYDV-MAV (Ueng et al, 1992). Au sous-groupe 2, représenté par le virus de l'enroulement de la pomme de terre (PLRV) (Mayo et al, 1989), sont associés le virus de la jaunisse occidentale de la betterave (BWYV) (Veidt et al, 1988), le BYDV-RPV (Vincent et al, 1991) et, récemment, le virus de la jaunisse des cucurbitacées transmis par puceron (CABYV) (Guilley et al, 1994). Un troisième sousgroupe, récemment proposé sur la base de l'organisation génomique du virus du nanisme du soja (SDV), serait intermédiaire entre les sousgroupes 1 et 2 (Rathjen et al, 1994).

Cinq ou 6 ORF ont été décrits chez les lutéovirus (fig 1). Les ORF 1 et 2 codent pour une putative ARN polymérase ARN-dépendante (RdRp). On a démontré chez certains membres des lutéovirus du sous-groupe 2, l'existence d'une 


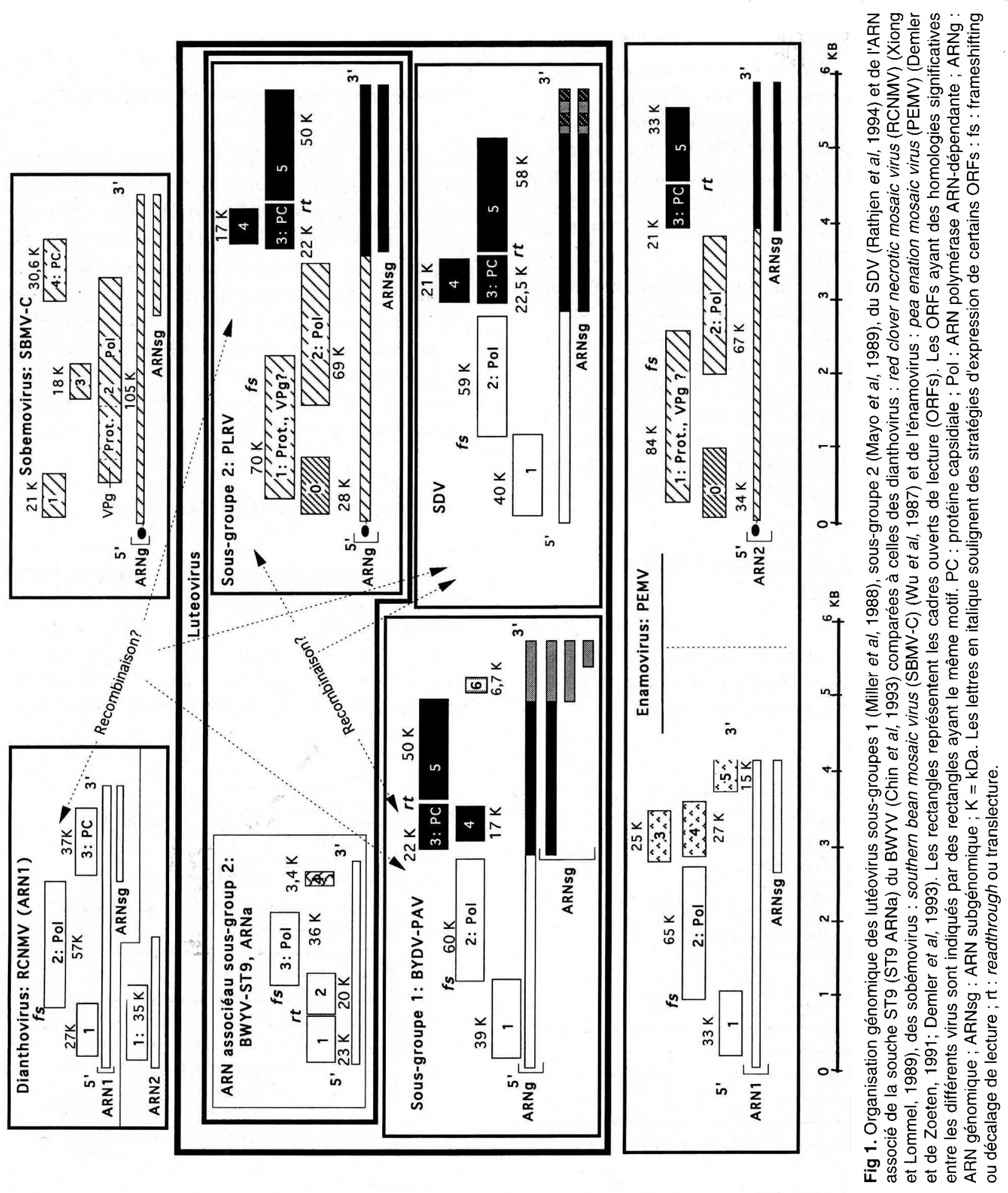


protéine liée d'une façon covalente à l'extrémité $5^{\prime}$ du génome ou VPg (virion protein genome-lin$k e d)$. Cette protéine serait codée par l'ORF1 qui présente aussi des motifs protéasiques (Miller et al, 1994). Les lutéovirus du sous-groupe 1 n'ont probablement pas de VPg (Miller et al, 1994 ; Young, commun pers).

L'ORF3 code pour la protéine capside (21$22 \mathrm{kDa}$ ) ; I'ORF4 code pour une protéine de 17 à $21 \mathrm{kDa}$ qui pourrait être impliquée dans le mouvement de cellule à cellule (Tacke et al, 1993). L'ORF5 code par translecture (suppression du codon stop de l'ORF3) pour une protéine structurale impliquée, avec la protéine capside, dans la transmission par puceron.

Un ORF supplémentaire (ORF0), codant pour une protéine de $28-29 \mathrm{kDa}$ et de fonction inconnue, est présent chez les lutéovirus du sousgroupe 2. En revanche, les lutéovirus du sousgroupe 1 présentent en plus, à l'extrémité 3'-terminale de leur génome, un petit ORF (ORF6) codant pour une protéine putative de 4,5 à $6,7 \mathrm{kDa}$ (Miller et al, 1988 ; Ueng et al, 1992). Le SDV ne possède ni l'ORF0, ni l'ORF6 (fig 1).

\section{DICHOTOMIE DANS LES RELATIONS PHYLOGÉNÉTIQUES}

Des phylogénies différentes sont observées entre les lutéovirus et d'autre phytovirus, en fonction de la région du génome considérée.

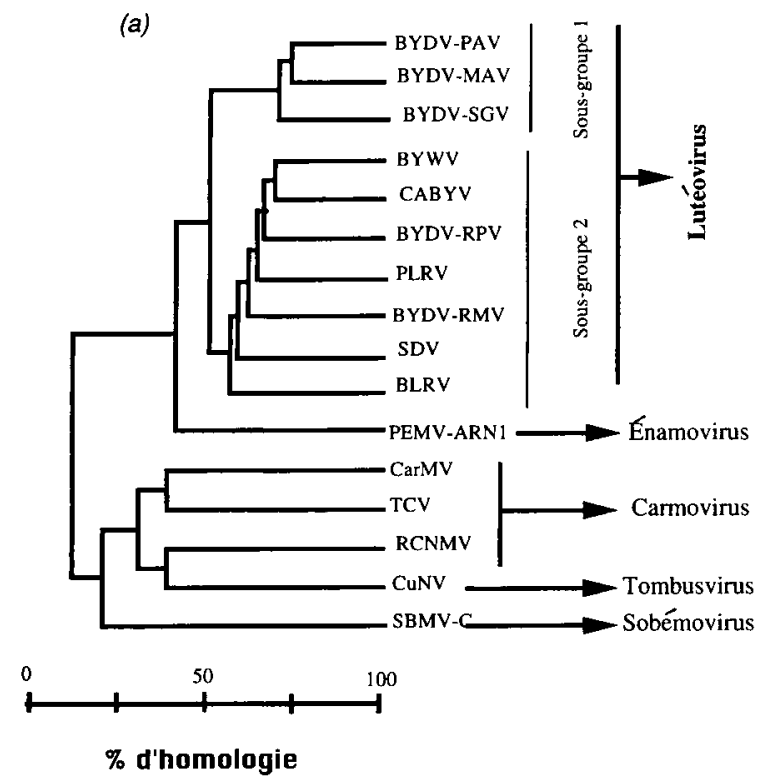

Pour préciser cette situation, il nous a paru intéressant d'analyser les données disponibles concernant les homologies de séquence de 2 parties représentatives du génome des lutéovirus : la protéine capside et la protéine de I'ARN polymérase (figs 1,2).

\section{Comparaison des séquences capsidiques}

Pour le gène capside, les homologies de séquence entre les différents lutéovirus sont plus grandes qu'avec les autres phytovirus. La figure $2 \mathrm{a}$, illustre, en fonction des homologies de séquences de la protéine capside, les liens existant entre les différents lutéovirus connus et ceux d'autres phytovirus. Le groupement des différents lutéovirus en fonction des homologies de séquence de la protéine capside (fig $2 a$ ) affine celui élaboré à partir des relations sérologiques (Waterhouse et al, 1988).

\section{Comparaison des ARN polymérases}

La comparaison des RdRp des différents lutéovirus et celles d'autres phytovirus (Habili et Symons, 1989 ; Martin et al, 1990 ; Koonin, 1991 ; Koonin et Dolja, 1993 ; fig 2b) montre que les ARN polymérases des lutéovirus du sous-groupe 1 et du SDV ont plus d'homologie

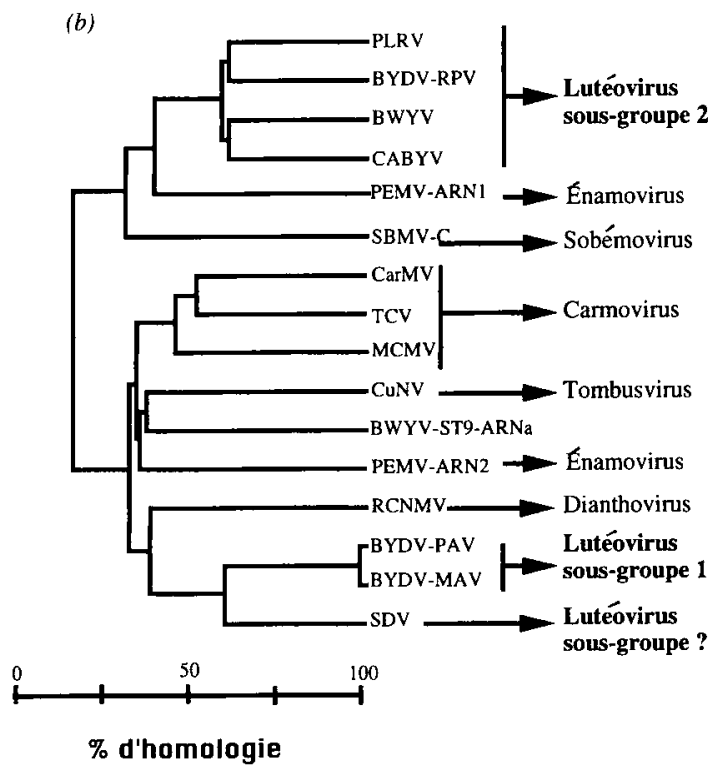

Fig 2. Comparaison des protéines capsides (a) et des polymérases (b) entre différents lutéovirus et d'autres phytovirus, retracée d'après les premières comparaisons de Martin et al (1990). Ont été ajoutés les virus suivants récemment séquencés : SDV (Rathjen et al, 1994) ; BYDV-RMV (Domier et al, 1994) ; BYDV-SGV (Lei et al, 1993) ; BYDV-RPV (Vincent et al, 1991), BYDV-MAV (Ueng et al, 1992) ; CABYV (Guilley et al, 1994) ; PEMV-ARN1 (Demler et De Zoeten, 1991) ; PEMV-ARN2 (Demler et al, 1993) ; BWYV-ST9 aRNA (Chin et al, 1993) ; BLRV (Prill et al, 1990). 
(environ 33\%) avec celles des carmovirus (carnation mottle virus: (arMV), tombusvirus (cucumber necrosis virus: CuNV), dianthovirus (red clover necrotic mosaic virus: RCNMV) qu'avec celles des lutéovirus du sous-groupe 2 ( $17 \%$ environ). En revanche, les RdRp des lutéovirus du sous-groupe 2 montrent plus d'homologie avec celles des sobémovirus (southern bean mosaic virus: SBMV) et de I'ARN1 de l'énamovirus : pea enation mosaic virus (PEMV) (fig 2b).

\section{RECOMBINAISONS ARN}

Plusieurs modèles peuvent être avancés pour expliquer la formation de cet semble de phytovirus interliés.

\section{Association d'ARNs taxonomiquement distincts}

Une association entre I'ARN d'un lutéovirus du sous-groupe 2 et un ARN portant une polymérase de type carmovirale, donc taxonomiquement distincts (fig 2b), est observée pour la souche ST9 et l'énamovirus PEMV.

Les 2 ARN génomiques du PEMV, I'ARN génomique et I'ARN associé au BWYV, sont capables de s'auto-répliquer.

Chez la souche ST9 du BWYV, I'ARN génomique (type lutéovirus du sous-groupe 2) ne dépend pas de son ARN associé pour induire une infection. L'ARN associé dépend de I'ARN génomique pour plusieurs fonctions comme l'encapsidation et le mouvement (Chin et al, 1993).

Les ARN 1 et 2 du PEMV constituent une association symbiotique. L'ARN1 dépend de I'ARN2 pour la transmission mécanique et aussi l'infection systémique (Demler et al, 1994), en raison de l'absence de l'ORF4 dans I'ARN1 (fig 1), impliqué probablement dans le mouvement de cellule à cellule (Take et al, 1993). En revanche, I'ARN2 dépend de I'ARN1 pour l'encapsidation et la transmission par puceron (Demler et al, 1993).

Cette association d'ARN constitue un bel exemple de «pseudo-recombinaison" qui a pu avoir lieu lors d'une infection mixte entre un virus de l'ordre des carmovirales et un lutéovirus du sous-groupe 2.

\section{Formation des lutéovirus des sous-groupes 1 et 2 par recombinaison $A R N$}

Les lutéovirus du sous-groupe 1 et le SDV pourraient avoir été générés par recombinaison entre la moitié $5^{\prime}$ de l'ARN1 d'un virus appartenant à l'ordre des carmovirales (un dianthovirus par exemple), codant pour la RdRp, et la moitié d'un lutéovirus du sous-groupe 2, codant pour les protéines structurales et du mouvement (Miller et al, 1994 ; fig 1). Cette recombinaison aurait pu avoir lieu lors d'infections mixtes, similaires à celles qui ont donné naissances aux associations d'ARN taxonomiquement distincts de type BWYV-ST9 ou PEMV.

Selon le même modèle, la région terminale du génome des lutéovirus du sous-groupe 1 , de 3 ' à I'ORF5, pourrait être issue d'un deuxième phénomène de recombinaison avec un ARN d'origine non encore identifiée (Miller et al, 1994).

D'autres modèles de recombinaison d'ARN peuvent être proposés à partir des comparaisons des séquences (fig 2) et des organisations génomiques (fig 1). Par exemple, on peut envisager que les lutéovirus du sous-groupe 2 aient été générés à partir d'une recombinaison lors d'une infection mixte entre un sobémovirus et un lutéovirus du sous-groupe 1 .

\section{Situation du SDV}

Le génome du SDV présente globalement plus d'homologie pour la moitié $3^{\prime}$ avec les lutéovirus du sous-groupe 2 (fig 2a) alors que la moitié $5^{\prime}$ est taxonomiquement proche de celles des lutéovirus du sous-groupe 1 (fig 2b). Cette situation a permis à Rathjen et al (1994) de proposer que le SDV serait issu d'une recombinaison entre la moitié 5 ' du génome d'un lutéovirus du sousgroupe 1 et la moitié $3^{\prime}$ du génome d'un lutéovirus du sous-groupe 2 .

Des zones conservées dans la région $3^{\prime}$-terminale des génomes du SDV et des lutéovirus du sous-groupe 1 (BYDV-PAV) ont été récemment identifiées (Chalhoub et al, 1994). Ces résultats rendent l'hypothèse de Rathjen et al (1994) contestable, à moins qu'une double recombinaison ait lieu aboutissant à l'insertion de la moitié $3^{\prime}$ codante d'un lutéovirus du sousgroupe 2 entre la moitié $5^{\prime}$ et la région $3^{\prime}$-terminale d'un lutéovirus du sous-groupe 1 (Chalhoub et al, 1994). 
Ces données, en revanche, renforcent l'hypothèse de la formation du SDV et des lutéovirus du sous-groupe 1 par recombinaison entre un dianthovirus et un lutéovirus du sous-groupe 2 (Miller et al, 1994). La moitié 3 ' des lutéovirus du sous-groupe 1 aurait rapidement divergé par rapport aux virus du sous-groupe 2 et du SDV grâce à des pressions de sélection différentes.

\section{Autres recombinaisons ARN impliquant les lutéovirus}

La comparaison de la protéine capside ainsi que les régions $\mathrm{N}$-terminale et $\mathrm{C}$-terminale de la protéine de translecture codées par l'ORF5, entre différents lutéovirus du sous-groupe 2, le SDV et l'énamovirus : PEMV, suggère un double événement de recombinaison entre les ancêtres du CABYV et du PEMV (Gibbs et Cooper, 1995). Ce phénomène de double recombinaison a abouti au transfert de l'ARN codant pour la région $\mathrm{N}$-terminale de la protéine de translecture de l'ancêtre du PEMV entre celui codant pour les protéines capsides et la région $\mathrm{C}$-terminale de l'ancêtre du CABYV.

D'autres phénomènes de recombinaisons seraient aussi produites entre différent isolats $\mathrm{du}$ BYDV-PAV, pour la région 3'-terminale du génome (Chalhoub et al, 1995).

Un phénomène de recombinaison entre I'ARN de l'hôte et la région 5 ' non codante d'une souche écossaise du PLRV (souche PLRV-S) a été décrit (Mayo et Jolly, 1991).

\section{CONSIDÉRATIONS ET PROPOSITIONS TAXONOMIQUES}

La comparaison des RdRp des virus à ARN simple brin de polarité positive est de plus en plus considérée comme un élément taxonomique fort (Habili et Symons, 1989 ; Koonin, 1991 ; Koonin et Dolja, 1993 ; Ward, 1993). Les premières comparaisons des motifs hélicases et polymérases ont aussi permis à Habili et Symons (1989) de diviser les lutéovirus en 2 sousgroupes distincts (1 et 2).

Avec la nécessité d'assigner les différents groupes de virus à des genres et familles comme le suggère l'ITCV, Martelli (1993) propose la définition du genre lutéovirus qui se divise en 2 types : type $A$, correspondant aux lutéovirus du sous-groupe 1 et type $B$, aux lutéovirus du sousgroupe 2.

Koonin et Dolja (1993) ont proposé l'appellation d'allolutéovirus pour les lutéovirus du sousgroupe 1 et de lutéovirus pour les lutéovirus du sous-groupe 2. Ces 2 genres appartiendraient respectivement aux ordres des carmovirales et sobémovirales. Ward (1993) a suggéré la constitution de 2 genres : les lutéovirus du sous-groupe 1 appartiendraient au genre des paralutéovirus et les lutéovirus du sous-groupe 2 au genre des ortholutéovirus. Cet auteur propose que ces 2 genres définissent les genres types des familles des Paraluteoviridae et des Ortholuteoviridae.

\section{DISCUSSION ET CONCLUSION}

Les infections mixtes qui peuvent avoir lieu entre les lutéovirus et d'autres phytovirus ont constitué probablement des situations favorables à une évolution vers de nouvelles entités virales. Ces nouveaux virus peuvent avoir des génomes composées d'ARN taxonomiquement distincts mais interdépendants, comme c'est le cas de la souche du BWYV-ST9 et du PEMV. Ils peuvent aussi avoir des génomes résultant de recombinaisons ARN selon les modèles présentés.

La dichotomie et la flexibilité apparente des moitiés $5^{\prime}$ et $3^{\prime}$ des génomes des lutéovirus constituent un exemple de recombinaison d'ARN aboutissant à la génération d'un ensemble de virus ayant des propriétés biologiques communes mais différant dans leur ARN réplicase (Koonin et Dolja, 1993 ; Miller et al, 1994). En effet, les lutéovirus constituent par leurs propriétés biologiques et, en partie par leurs propriétés sérologiques, un groupe homogène. Les données cytopathologiques (Gill et Chong, 1979) et celles établies par sondes nucléiques (Waterhouse et al, 1986 ; Fattouh et al, 1990), permettent de les diviser en 2 grands sousgroupes. La comparaison de l'organisation du génome de plusieurs virus de ce groupe montre qu'ils ont plus d'homologies entre eux, pour les ORF responsables de certaines propriétés biologiques et sérologiques (codées par la moitié $3^{\prime}$ du génome), qu'avec les virus des autres groupes. C'est aussi dans cette partie du génome que s'effectue la translecture soupçonnée d'être responsable de la localisation des lutéovirus au phloème de la plante hôte (Rohde et al, 1993). En revanche, ils divergent dans la partie $5^{\prime}$ de leur génome codant, en particulier, pour les RdRp. 
II est intéressant de noter que les sites des recombinaisons ARN, proposées pour expliquer la génération des différents lutéovirus, coïncident avec la présence d'ARN subgénomiques (Miller et al, 1994 ; Chalhoub et al, 1995 ; fig 1). Ces ARN subgénomiques pourraient présenter à leur extrémité $5^{\prime}$ des séquences ou des structures dont l'accès, plus facile pour la réplicase virale, faciliterait les phénomènes de recombinaison (Lai, 1992 ; Millet et al, 1994).

La dichotomie entre les moitiés $5^{\prime}$ et $3^{\prime}$ du génome des lutéovirus justifie certainement que leur classification soit construite avec davantage d'éléments objectifs. Les propositions taxonomiques doivent tenir compte des arguments suivants : i) considérer les 2 sous-groupes comme membres de la même sous-famille ou famille n'est pas souhaitable, en raison des différences majeures concernant les ARN polymérases ; ii) considérer que les lutéovirus du sous-groupe 1 (et le SDV) constituent un genre de la famille des Tombusviridae ou des Dianthoviridae et ceux du sous-groupe 2 un genre de la famille des Sobemoviridae sur la base de la taxonomie de leur RdRp uniquement n'est pas davantage souhaitable, en raison des ressemblances entre les 2 sous-groupes concernant la moitié 3 ' de leur génome et les différences avec les Sobemoviridae et Tombusviridae dans cette même partie du génome (fig $2 b$ ).

Par ailleurs, la définition et l'appellation des 2 genres (paralutéovirus et ortholutéovirus), membres des familles des Paraluteoviridae et Ortholuteoviridae (Ward, 1993), peuvent entraîner des confusions. En particulier, la signification donnée aux préfixes «para-» et «ortho-» dans ce contexte est prématurée. Enfin, actuellement, bien que toute tentative de classification des virus reste subjective, on ne peut s'empêcher de proposer une terminologie en tenant compte des relations observées entre les $\operatorname{RdRp}$ de ces virus et celles d'autres groupes. Le genre carmolutéovirus (de carmovirale) pourrait correspondre aux lutéovirus du sous-groupe 1 ; le genre sobémolutéovirus (de sobémovirale) pourrait correspondre aux lutéovirus du sous-groupe 2 .

\section{RÉFÉRENCES}

Casper R (1988) Luteoviruses. In : The Plant Viruses: Polyhedral Virions with Monopartite RNA Genomes (R Koenig, ed), Plenum Press, New York, Vol 3, 235-258

Chalhoub BA, Kelly L, Robaglia C, Lapierre HD (1994) Evidence of RNA recombination in the genome $3^{\prime}-$ terminal region of PAV-like isolates of barley yellow dwarf virus (BYDV-PAV). agronomie 15, 409-413

Chalhoub BA, Kelly L, Robaglia C, Lapierre HD (1994) Sequence variability in the genome-3'-terminal region for 10 geographically distinct PAV-like isolates of barley yellow dwarf virus: analysis of the ORF6 variation. Arch Virol 139, 403-416

Chin LS, Foster JL, Falk BW (1993) The beet western yellows virus ST9-Associated RNA shares structural and nucleotide sequence homology with Carmo-like viruses. Virology 192, 473-482

Demler SA, Borkhsenious ON, Rucker DG, de Zoeten GA (1994) Assessment of the autonomy replicative and structural functions encoded by the luteo-phase of pea enation mosaic virus. J Gen Virol 75, 9971007

Demler SA, de Zoeten GA (1991) The nucleotide sequence and luteovirus-like nature of RNA 1 of an aphid non-transmissible strain of pea enation mosaic virus. J Gen Virol 72, 1819-1834

Demler SA, Rucker DG, de Zoeten GA (1993) The chimeric nature of the genome of pea enation mosaic virus: the independent replication of RNA 2.J Gen Virol 74, 1-14

Domier LL, Lukasheva LI, D'Arcy CJ (1994) Coat protein sequences of RMV-like strains of barley dwarf virus separate them from other luteoviruses. Intervirology 37, 2-5

Duffus JE, Falk BW, Johnstone GR (1990) Luteoviruses-one system, many variations. In : World Perspective on Barley Yellow Dwarf (PA Burnett, ed), CIMMYT, Mexico, 86-104

Fattouh FA, Ueng PP, Kawata EE, Barbara DJ, Larkins BA, Lister RM (1990) Luteovirus relationships assessed by cDNA clones from barley yellow dwarf viruses. Phytopathology 80, 913-920

Gibbs MJ, Cooper Jl (1995) A recombinational event in the history of luteoviruses probably induced by base-paring between the genomes of 2 distinct viruses. Virology 206, 1129-1132

Gill CC, Chong J (1975) Development of the infection in oat leaves inoculated with barley yellow dwarf virus. Virology $66,440-453$

Gill CC, Chong J (1979) Cytopathological evidence for the division of barley yellow dwarf virus isolates into 2 subgroups. Virology 95, 59-69

Guilley H, Wipf-Sheibel C, Richards K, Lecoq H, Jonard G (1994) Nucleotide sequence of cucurbit aphid-borne yellow luteovirus. Virology 202, 10121017

Habili N, Symons RH (1989) Evolutionary relationship between luteoviruses and other RNA plant viruses based on sequence motifs in their putative RNA dependent RNA polymerases and nucleic acid helicases. Nucleic Acids Res 17, 9543-9555

Johnstone GR, Sward RJ, Farrell JA et al (1990) Epidemiology and control of barley yellow dwarf viruses in Australia and New-Zealand. In : World Perspectives on Barley Yellow Dwarf (PA Burnett, ed), CIMMYT, Mexico, 228-237 
Koonin E (1991) The phylogeny of RNA-dependent RNA polymerases of positive-strand RNA viruses. $J$ Gen Virol 72, 2197-2206

Koonin EV, Dolja VV (1993) Evolution and taxonomy of positive-strand RNA viruses: Implications of comparative analysis of amino-acid sequences. Crit Rev Biochem Molec Biol 28, 375-430

Lei $\mathrm{CH}$, Lister RM, Vincent JR (1993) Relationships of 2 SGV serotype isolates with other barley yellow dwarf viruses. IXth Intern Congr Virol, Glasgow 354

Martelli GP (1993) The new classification of plant viruses. Petria 3, 131-140

Martin RR, Keese PK, Young MJ, Waterhouse PM, Gerlach WL (1990) Evolution and molecular biology of luteoviruses. Annu Rev Phytopathol 28, 341-363

Matthews REF (1982) Classification and nomenclature of viruses. Rep Int Comm Taxonomy Viruses. 4th Intervirology 17, 3-181

Mayo MA, Jolly CA (1991) The 5'-terminal sequence of potato leafroll virus RNA: evidence of recombination between virus and host RNA. J Gen Virol 72, 25912595

Mayo MA, Robinson CA, Jolly CA, Hyman L (1989) Nucleotide sequence of potato leafroll luteovirus RNA. J Gen Virol 70, 1037-1051

Miller WA, Dinesh-Kumar SP, Paul CP (1995) Luteovirus gene expression. Critical Reviews in Plant Science 14, 179-211

Miller WA, Waterhouse PM, Gerlach WL (1988) Sequence and organization of barley yellow dwarf virus genomic RNA. Nucleic Acids Res 16, 6097 . 6111

Palasingam K, Shaklee PN (1992) Reversion of QB RNA phage mutants by homologous RNA recombination. J Viro/ 66, 2435-2442

Prill B, Maiss E, Katul L, Casper R (1990) Nucleotide sequence of the bean leafroll luteovirus coat protein gene. Nucleic Acids Res 18, 5544

Rathjen JP, Karageorgod LE, Habili N, Waterhouse PM, Symons RH (1994) Soybean dwarf luteovirus contains the third variant genome type in the luteovirus group. Virology 198, 671-679

Rochow WF, Duffus JE (1981) Luteoviruses and yellows diseases. In: Handbokook Plant Virus Infection
Comparative Diagnosis (E Kurstak, ed), NorthHolland, Amsterdam, 147-170

Rohde W, Prüfer D, Schmitz J, Tacke E, Randles JW (1993) Factors that regulate the phloem-specific replication of the luteovirus PLRV and the 'circovirus' CFDV. IXth Intern Congr Virol Glasgow, 328

Shephered RJ, Franki RI, Hirth L et al (1976) New groups of plant viruses approved by the International Committee on Taxonomy of Viruses. Intervirology 6, 181-184

Simon AE, Bujarski JJ (1994) RNA recombination and evolution in virus-infected plants. Annu Rev Phytopathol 32, 337-362

Tacke E, Schmitz J, Prüfer D, Rohde W (1993) Mutational analysis of the nucleic acid-binding $17 \mathrm{kDa}$ phosphoprotein of potato leafroll luteovirus identifies an amphipathic $\alpha$-helix as the domain for protein/protein interactions. Virology 197, 274-282

Ueng PP, Vincent JR, Kawata EE, Lei CH, Lister RM, Larkins BA (1992) Nucleotide sequence analysis of the genomes of the MAV-PS1 and P-PAV isolates of barley yellow dwarf virus. J Gen Virol 73, 487492

Veidt I, Lot H, Lieiser $M$ et al (1988) Nucleotide sequence of beet western yellows. Nucleic Acids Res 16, 9917-9932

Vincent JR, Lister RM, Larkins BA (1991) Nucleotide sequence analysis and genomic organization of the NY-RPV isolate of barley yellow dwarf virus. $J$ Gen Virol 72, 2347-2355

Ward CW (1993) Progress towards a higher taxonomy of viruses. Res Virol 144, 419-453

Waterhouse PM, Gerlach WL, Miller WA (1986) Serotype-specific and general luteovirus probes from cloned cDNA sequences of barley yellow dwarf virus. J Gen Virol 67, 1273-1281

Waterhouse PM, Gildow FE, Johnstone GR (1988) Luteovirus group. In : CMI: Assoc Appl Biol Description of Plant Viruses 339, Wellesbourne, Warwick, UK

Wu S, Riehart C, Kaesberg P (1987) Sequence and organization of southern bean mosaic virus genomic RNA. Virology 161, 73-80

Xiong Z, Lommel SA (1989) The complete nucleotide sequence and genome organization of red clover necrotic mosaic virus RNA-1. Virology 171, 543-554 